\title{
Originales
}

\section{El examen de seis músculos individuales: ¿Puede representar un enfoque diagnóstico de trastornos neuropáticos de los miembros superiores?}

\section{Can testing of six individual muscles represent a screening approach to upper limb neuropathic conditions?}

\section{Jørgen Riis Jepsen 1,2 Jose Hernán Alfonso ${ }^{3,4}$}

1. Departamento de Medicina Laboral, Hospital de South-western Jutland. Østergade 81-83, Esbjerg DK-6700, Dinamarca.

2. Centre of Maritime Health and Society, University of Southern Denmark, Niels Bohrs Vej 9-10, Esbjerg DK-6700, Dinamarca.

3. Departamento de Medicina Ocupacional y Ambiental. Hospital Universitario de Oslo. Noruega.

4. Departamento de Medicina Ocupacional y Epidemiologia. Instituto Nacional de Salud Ocupacional. Noruega.

Recibido: 13-11-14

Aceptado: 18-11-14

\section{Correspondencia}

Jose Hernán Alfonso

Correo electrónico: jose.alfonso@stami.no

(C) 2014 Jepsen; licensee BioMed Central Ltd. Este artículo ha sido publicado en inglés en

BMC Neurology 2014, 14:90 La versión en inglés se halla disponible online en http://

www.biomedcentral.com/1471-2377/14/90

El primer autor cuenta con el Copryright del articulo; su uso, distribución y reproducción están

permitidos siempre que se reconozca el trabajo original (términos de Creative Commons

AttributionLicense (http://creativecommons.org/licenses/by/2.0)

La adaptación a la lengua castellana fue realizada por el segundo autor y el contenido

médico científico del mismo es fiel al artículo original.

Las experiencias clínicas aquí mencionadas pertenecen al Departamento de Medicina

Laboral de Esbjerg, Dinamarca.

Resumen

Antecedentes: Se ha demostrado anteriormente que un examen neurológico exhaustivo de la función muscular individual de los miembros superiores, de la sensación en zonas inervadas homónimas y la alodinia en nervios y tronco es confiable y el resultado refleja los síntomas. Debido a que este enfoque puede parecer complicado y que requiere mucho tiempo, este estudio se ocupa de la validez de un examen que se limita al chequeo manual de sólo seis músculos.

Métodos: Dos examinadores cegados al estado de los síntomas realizaron exámenes musculares de manera manual de seis músculos en 82 miembros superiores con o sin dolor, debilidad y/o entumecimiento/ hormigueo. Los seis músculos representan tres pares antagonistas (pectoral mayor/ deltoideo posterior, bíceps/tríceps y flexor radial de la muñeca/extensor radial corto de la muñeca). La fiabilidad entre calificadores para detectar debilidades musculares y la relación de la debilidad con los síntomas mencionados se analizaron por medio de la analisis kappa.

Resultados: Los dos examinadores reconocieron debilidad en 48 y 55 miembros respectivamente, con una coincidencia moderada (valor mediano de kappa = 0,58). De estos, 35 y 32 miembros respectivamente fueron sintomáticos. Hubo una buena correlación entre los hallazgos y los síntomas para un examinador 
(kappa $=0,61)$ y una correlación justa para el otro (kappa =0,33). La sensibilidad fue alta $(0,92$ y 0,84$)$ para ambos, pero la especificidad menos satisfactoria $(0,70$ y 0,50$)$. La debilidad tuvo una correlación moderada con los síntomas (kappa $=0,57)$ para los dos examinadores.

Conclusiones: Debilidad muscular en uno o más músculos estuvo presente en casi todos los miembros sintomáticos pero también en muchos miembros asintomáticos. El examen manual de seis músculos puede representar un enfoque diagnóstico para los trastornos neuropáticos de miembros superiores pero un diagnóstico confirmativo requiere más evaluaciones.

Med Segur Trab (Internet) 2014; 60 (237) 627-644

Palabras clave: Examen neurológico, examen muscular, trastornos neuropáticos, miembros superiores, debilidad muscular, enfermedades ocupacionales.

Abstract

Background: It has previously been demonstrated that an extensive upper limb neurological examination of individual muscle function, sensation in homonymous innervated territories, and nerve trunk allodynia is reliable and that the outcome reflects symptoms. Since this approach may appear complicated and time consuming, this study deals with the value of an examination limited to manual testing of only six muscles.

Methods: Two examiners blinded to symptom status performed manual muscle testing of six muscles in 82 upper limbs with or without pain, weakness, and/or numbness/tingling. The six muscles represent three antagonist pairs (pectoralis major/posterior deltoid, biceps/triceps, and radial flexor of wrist/short radial extensor of wrist). The inter-rater reliability of detecting muscular weaknesses and the relation of weakness to the mentioned symptoms were analysed by kappa-statistics.

Results: The two examiners recognized weaknesses in 48 and 55 limbs, respectively, with moderate agreement (median kappa $=0.58$ ). Out of these, 35 and 32 limbs, respectively, were symptomatic. There was good correlation between findings and symptoms for one examiner (kappa $=0.61$ ) and fair correlation for the other one (kappa $=0.33)$. Both reached high sensitivity $(0.92,0.84)$ but less satisfactory specificity $(0.70,0.50)$. Weaknesses agreed upon by the two examiners correlated moderately with symptoms (kappa $=0.57)$.

Conclusions: Weakness in one or more muscles was present in almost all symptomatic limbs but in many non-symptomatic limbs as well. Manual testing of six muscles may represent a useful screening approach to upper limb neuropathic conditions, but a confirmative diagnosis requires further assessment.

Med Segur Trab (Internet) 2014; 60 (237) 627-644

Key Words: neurological examination, muscle testing, upper limb pain, muscle weakness, neuropathic disorders, occupational diseases 


\section{INTRODUCCIÓN}

La alta prevalencia de los trastornos en los miembros superiores con relación al trabajo, sus efectos en la calidad de vida y capacidad en el trabajo y el progreso limitado en relación al diagnóstico, manejo y prevención requieren nuevas perspectivas en este campo de investigación y práctica ${ }^{1,2}$.

En particular, hay una clara necesidad de consenso en relación a los exámenes físicos y criterios de diagnóstico de una eficacia diagnóstica suficiente. Palmer y Cooper estiman que un enfoque físico convencional permite la clasificación diagnóstica de solo un cuarto de pacientes con trastornos en las extremidades superiores con relación al trabajo ${ }^{3}$.

Se considera a menudo que los pacientes restantes sufren de los llamados trastornos «no específicos» que pueden precisarse, por ej. «síndrome por esfuerzo de repetición» que indica un estado de patofisiologia poco clara y que se lo asocia con exposiciones adversas de trabajo físico. Aún con la ausencia de pruebas de apoyo en términos de hallazgos físicos, hay también una tendencia a diagnosticar a muchos de los pacientes con trastornos en las extremidades superiores de acuerdo a la ubicación dominante de los síntomas, por ejemplo, epicondilitis con dolor en el codo o trastorno del manguito rotador con dolor en el hombro. Dichos diagnósticos, sin embargo, no reflejan el tipo de tejido lesionado, la localización ni la patología implicada. Si los síntomas han de considerarse de naturaleza neuropática, es probable que estos pacientes sean sometidos a estudios electrofisiológicos o de imagen en lugar de un examen exhaustivo de las funciones de los nervios periféricos de miembros superiores.

El valor de las definiciones de casos (criterios de diagnóstico que consisten en una combinación de síntomas y señales que caracterizan determinado trastorno) está en la utilidad práctica de distinguir grupos de personas con los mismos síntomas y/o características físicas o cuya enfermedad comparta las mismas causas o determinantes de resultado. Por lo tanto, la mejor definición de caso diagnóstico para un trastorno puede variar de acuerdo al propósito, por ejemplo, epidemiológico o clínico para la cual está siendo aplicada ${ }^{4}$. Aún así, la definición de caso debe reflejar el tejido lesionado y su ubicación.

Muchos de los pacientes con trastornos en miembros superiores presentan una tríada de síntomas que consisten en debilidad, entumecimiento/hormigueo y dolor, frecuentemente de carácter neuropático. Estos síntomas sugieren una implicación de los nervios periféricos. Los patrones de anomalías neurológicas definidos por la ramificación e inervación nerviosa (debilidad muscular selectiva ${ }^{5}$, anomalías sensoriales y alodinia mecánica de los troncos nerviosos) pueden reflejar una neuropatía focal en miembros superiores con localizaciones específicas.

Esto resultó en el desarrollo y la validación de una evaluación neurológica exhaustiva, sistemática y detallada de miembros superiores con el objetivo de complementar el examen físico convencional en pacientes derivados a un departamento de medicina laboral. El examen neurológico esta basado en la probabilidad de una incapacidad en funciones motrices y sensoriales distales a un segmento atrapado de un nervio que inerva y en el hecho de que el nervio va a presentar un dolor anormal al momento de palparlo en la localización del atrapamiento.

Se ha demostrado que este examen es confiable y capaz de descubrir la presencia de patrones neurológicos en miembros superiores sintomáticos y su ausencia en miembros asintomáticos $^{6-8}$. En consecuencia, esta evaluación neurológica puede ser significativa, en particular cuando falla el examen físico convencional para identificar anomalías. Este fue el caso en 13 de 16 miembros sintomáticos que de otro modo, no podrían haberse caracterizado diagnósticamente, es decir, «dolor de brazo no específico» 8 .

Los clínicos que están menos familiarizados con las propiedades biomecánicas de los músculos y con el curso de los nervios de las extremidades superiores y su inervación muscular y cutánea pueden considerar dicho examen neurológico exhaustivo ${ }^{6,7}$ como un examen que requiere mucho tiempo, difícil de realizar e interpretar. Por lo tanto, un 
método de evaluación diagnostico simple en los nervios de las extremidades superiores sería de importancia. En la reunión de 1993 de La Sociedad Escandinava para la Cirugía de Mano, Hagert presentó un examen basado en el chequeo manual del músculo de seis músculos que fueron seleccionados de 60 músculos del hombro y miembros superiores para reflejar la neuropatía focal con localizaciones específicas (pectoral mayor/deltoideo posterior, bíceps/tríceps y flexor radial de la muñeca/extensor radial corto de la muñeca). Este examen fue desarrollado para reflejar neuropatía focal con localizaciones específicas. Concluyó que una aflicción en la ramificación nerviosa de los miembros superiores era improbable con fuerza normal en estos músculos así como también en el abductor pequeño del dedo meñique, el abductor pequeño del dedo pulgar y el extensor ulnar de la muñeca. Una publicación más reciente brinda una descripción detallada de la técnica para el examen de ocho músculos que son representativos de los nervios de los miembros superiores y de la interpretación de los resultados 5 .

Los seis músculos que Hagert (Tabla I) sugiere, fueron seleccionados para este estudio porque son simples de recordar y examinar. Además, son razonablemente representativos de cuatro (de las cinco raíces cervicales que forman el plexo braquial (C5, C6, C7 y C8) (Figura 1) así como también del plexo braquial y la mayoría de los nervios individuales de los miembros superiores. Por lo tanto, es probable que uno o más de estos músculos estén implicados en muchas afecciones de los nervios de los miembros superiores. La debilidad en estos músculos puede reflejar neuropatía focal periférica y, de acuerdo a la experiencia, es un hallazgo común si se lo busca.

Este estudio tiene como objetivo aclarar dos preguntas que son cruciales para evaluar en qué medida un examen limitado es un enfoque inicial físico viable en los nervios de los miembros superiores:

¿Es posible identificar de manera confiable la debilidad en los seis músculos seleccionados de miembros superiores?

La presencia de debilidad en cualquiera de estos seis músculos ¿tiene correlación con las quejas de los pacientes en cuanto a dolor, debilidad y/o entumecimiento/ hormigueo?

\section{MÉTODOS}

\section{Pacientes}

Los pacientes que participaron eran idénticos a aquellos en la serie previamente estudiada de 41 pacientes derivados consecutivamente: 22 pacientes de sexo masculino con edad mediana de 44 años (rango 29-61) y 19 pacientes de sexo femenino con edad mediana de 39 años (rango 25-52). Antes del examen, los pacientes del estudio fueron seleccionados de acuerdo a criterios definidos entre pacientes con cualquier tipo de trastorno (limitado o no a los miembros superiores) derivados al Departamento de Medicina Laboral, Hospital South-western Jutland, Esberg. Diecisiete pacientes fueron excluidos porque se sabía por contactos anteriores, que tenían problemas de comunicación, que habían pasado anteriormente por cirugía de miembros superiores o porque su apariencia sugería fácilmente que padecían una enfermedad tal como asma o enfermedad lumbar incapacitante. Quince pacientes se negaron a participar. Por razones de capacidad (máximo un paciente en estudio por día), diez pacientes comparables con los pacientes del estudio con respecto al patrón de enfermedad y gravedad, también fueron excluidos.

Veintidós pacientes fueron derivados debido a quejas acerca de un miembro y cinco pacientes debido a quejas acerca de ambas extremidades superiores. De nueve pacientes derivados por razones no relacionadas con quejas acerca de los miembros superiores, seis pacientes presentaron quejas y tres pacientes se habían quejado acerca de los miembros superiores. Cinco pacientes no presentaban quejas actuales o previas acerca de los miembros superiores ${ }^{6-8}$. 
Los pacientes representaban un amplio espectro de enfermedades con respecto a la gravedad y la duración. Fueron derivados preferentemente para evaluar las probabilidades de que el trastorno estuviese relacionado con el trabajo y las consecuencias para la vida laboral futura. Los pacientes que presentaron quejas acerca de los miembros superiores fueron derivados sin diagnóstico específico o se interpretó que sus quejas estaban relacionadas con condiciones tales como trastorno del manguito rotador, epicondilitis o síndrome del túnel carpiano. Por lo tanto, los síntomas pueden o no estar relacionados con un trastorno neurológico. El diagnóstico de interconsulta de los pacientes que no fueron derivados debido a quejas de los miembros superiores fueron por ejemplo: dermatitis o asma.

El estudio cumplió con la Declaración de Helsinki. Fue aprobado por el Comité de Ética (De Videnskabsetiske Komitéer for Region Syddanmark) y se obtuvo la firma del consentimiento informado de todos los participantes.

\section{Fiabilidad entre calificadores}

Todos los pacientes se sometieron a idénticas evaluaciones neurológicas de cabecera por parte de dos examinadores. No hubo comunicación entre los dos examinadores. Los exámenes tuvieron lugar en salas separadas y se llevaron a cabo uno inmediatamente después del otro. Ambos examinadores desconocían completamente las características de los pacientes y la comunicación con ellos se limitó a instrucciones relacionadas con el examen. Mientras el examen consistió en los puntos informados anteriormente (14 músculos individuales así como también alodinia mecánica y sensorial de los troncos nerviosos en localizaciones definidas ${ }^{6,7}$ ), el estudio actual sólo evaluó el resultado de la prueba manual de seis músculos individuales de miembros superiores (en ambos lados) que representan tres pares antagonistas (Tabla I).

Tabla I. Prueba manual de tres pares de músculos antagonistas en 82 extremidades superiores

\begin{tabular}{|c|c|c|c|c|c|c|c|}
\hline \multirow{2}{*}{$\begin{array}{c}\text { Par } \\
\text { músculo } \\
\text { antagonista }\end{array}$} & \multirow[b]{2}{*}{ Músculo } & \multirow[b]{2}{*}{ Nervio } & \multicolumn{2}{|c|}{ Debilidad } & \multirow{2}{*}{$\begin{array}{l}\text { Concordancia } \\
\text { relativa } \%[6]\end{array}$} & \multirow{2}{*}{$\begin{array}{c}\text { Valor-kappa } \\
\text { (intervalos de } \\
\text { confianza) } \\
\text { [6] }\end{array}$} & \multirow{2}{*}{$\begin{array}{c}\text { Posición de salida para la } \\
\text { prueba muscular } \\
{[6]}\end{array}$} \\
\hline & & & $\begin{array}{c}\text { Exam. } \\
1\end{array}$ & $\begin{array}{c}\text { Exam. } \\
2\end{array}$ & & & \\
\hline \multirow[t]{2}{*}{ I } & $\begin{array}{l}\text { Pectorales } \\
\text { mayores }\end{array}$ & Pectorales & 16 & 21 & 84 & $0.55(0.34-0.76)$ & \multirow{2}{*}{$\begin{array}{l}90 \text { grados flexión } \\
\text { hombro. } \\
\text { Extremidades superiores } \\
\text { puestas horizontalmente, } \\
\text { antebrazos en pronación. } \\
\text { (Figuras } 2 \text { y 3) }\end{array}$} \\
\hline & $\begin{array}{l}\text { Deltoideo } \\
\text { posterior }\end{array}$ & Axilar & 48 & 50 & 80 & $0.59(0.42-0.77)$ & \\
\hline \multirow[t]{2}{*}{ II } & $\begin{array}{l}\text { Bíceps } \\
\text { braquial }\end{array}$ & Musculocutáneo & 36 & 31 & 79 & $0.57(0.40-0.75$ & \multirow{2}{*}{$\begin{array}{l}90 \text { grados flexión del } \\
\text { codo. Parte superior de } \\
\text { brazos verticalmente } \\
\text { contra laterales del pecho } \\
\text { y antebrazos horizontales } \\
\text { La función supinadora } \\
\text { del bíceps también puede } \\
\text { testearse. (Figuras } 4 \text { y } 5 \text { ) }\end{array}$} \\
\hline & Trícepsbraquial & Radial & 34 & 33 & 87 & $0.72(0.57-0.88)$ & \\
\hline \multirow[t]{2}{*}{ III } & $\begin{array}{l}\text { Flexor radial } \\
\text { de la muñeca }\end{array}$ & Mediano & 32 & 32 & 77 & $0.46(0.25-0.66)$ & \multirow[b]{2}{*}{$\begin{array}{l}90 \text { grados flexión } \\
\text { del codo. Antebrazos } \\
\text { descansan sobre muslos: } \\
\text { para la prueba del flexor } \\
\text { radial de la muñeca, los } \\
\text { antebrazos en supinación } \\
\text { y dedos flexionados. Para } \\
\text { la prueba del extensor } \\
\text { radial corto de la muñeca } \\
\text { antebrazos en pronación } \\
\text { y dedos extendidos } \\
\text { (Figuras } 6 \text { y 7) }\end{array}$} \\
\hline & $\begin{array}{l}\text { Extensor radial } \\
\text { corto de la } \\
\text { muñeca }\end{array}$ & Radial & 29 & 20 & 84 & $0.69(0.53-0.85)$ & \\
\hline
\end{tabular}


Las habilidades técnicas en el procedimiento manual de prueba de fuerza muscular son cruciales. Se necesita algo de experiencia para realizar el examen correctamente y para interpretar si la fuerza es normal o reducida. Puede resultar difícil de evaluar la fuerza levemente reducida. Ambos examinadores habían aprendido la técnica de examinación poco tiempo antes del estudio. Un examinador la había utilizado durante dos años antes del estudio y el otro durante solo dos meses para seguir una actualización de la anatomía de miembro superior y la examinación supervisada de 20 pacientes.

Figura 1. Raíces, plexo braquial y nervios periféricos de miembros superiores. Esquema e inervación de los seis músculos seleccionados de miembros superiores

\begin{tabular}{|c|c|c|c|c|}
\hline C5 & C6 & C7 & C8 & Th1 \\
\hline \multicolumn{2}{|c|}{ Deltoide } & & & \\
\hline \multicolumn{2}{|c|}{ Biceps braquial } & & & \\
\hline & & \multicolumn{3}{|c|}{ Pectoral } \\
\hline & & ECRB & \\
\hline & & FCR & \\
\hline & \multicolumn{3}{|c|}{ Triceps } & \\
\hline
\end{tabular}

\section{Procedimiento para la prueba manual, semi cuantitativa, isométrica del músculo}

Cada par antagonista de músculos fue examinado estrictamente de manera sistemática desde los dos músculos proximales hasta los dos músculos distales. El lado derecho e izquierdo fueron evaluados simultáneamente con la extremidad posicionada de manera que optimizara la función aislada de las extremidades examinadas en particular (Tabla I) ${ }^{6,10}$.

Los brazos del paciente estaban elevados horizontalmente hacia adelante manteniendo los codos completamente extendidos, los antebrazos en pronación, las muñecas en posición neutral y la mano apretada. El examinador de pie delante del paciente, la aducción del brazo (músculos pectorales) y abducción (deltoideo posterior) fueron examinados aplicando fuerza contra las muñecas del paciente desde adentro hacia afuera y desde afuera hacia adentro respectivamente (Figuras 2 y 3 respectivamente). La posición preferida de salida para el deltoideo posterior es hacer que el paciente mantenga los brazos 30 grados hacia afuera.

II. La parte superior de los brazos del paciente ahora se mantienen a los costados del pecho, los codos flexionados en ángulo recto y apoyados contra el respaldo de la silla, los antebrazos apuntando hacia adelante manteniéndose en posición neutral, las muñecas en posición neutral y manos cerradas. El examinador, de pie delante del paciente, se inclina hacia adelante y hacia las muñecas del paciente, pidiéndole que lo "cargue" (flexión del codo, definido como bíceps) (Figura 4). Finalmente, de pie detrás del paciente, el examinador levanta las muñecas del paciente hacia arriba (tríceps) contra la resistencia del paciente (Figura 5).

III. El paciente se inclina hacia adelante descansando los antebrazos en los muslos con las muñecas apenas lejos de las rodillas. El paciente mantiene los antebrazos en supinación completa, las manos cerradas y las muñecas ligeramente flexionadas y el examinador se inclina hacia adelante y presiona la articulación interfalángica proximal de los nudillos de los dedos índice y medio con el fin de extender las muñecas del paciente (FCR) (Figura 6). El paciente mantiene los antebrazos en pronación completa, las manos abiertas y las muñecas extendidas y el examinador se inclina hacia adelante y presiona contra los nudillos de los dedos índice y medio con el fin de flexionar las muñecas del paciente (ECRB) (Figura 7).

Se pueden aplicar varios sistemas de clasificación para la evaluación de la función muscular. Este estudio, así como también el índice de Motricidad y la Puntuación Motora utilizan el sistema de clasificación del Consejo de Investigaciones Médicas ${ }^{11}$, y todos estos 
sistemas de clasificación buscan caracterizar el estado usando un número limitado de músculos.

Ambos examinadores clasificaron cada extremidad con respecto tanto a la presencia de debilidad definida como grado 4+ o menos en cualquiera de los seis músculos en la Tabla I o como a la fuerza intacta en los seis músculos.

\section{Validez de constructo}

La validez de constructo de la evaluación puede estudiarse examinando si las medidas de constructos que teóricamente deben o no deben estar relacionadas unas con otras, están, en realidad, relacionadas. La debilidad muscular, que es causada por una aflicción neurológica como el atrapamiento de nervios, es probablemente sintomática (validez convergente), mientras que los síntomas son menos probables en las extremidades sin debilidad (validez discriminante). Por lo tanto, la presencia o ausencia de debilidad(es) se comparó con la presencia o ausencia de quejas relacionadas con miembros superiores (dolor, debilidad y/o entumecimiento/hormigueo). Dos entrevistadores que no se comunicaron con los examinadores mencionados anteriormente, reunieron información acerca de las quejas de los pacientes con respecto a los miembros superiores ${ }^{8}$.

Figura 2. Postura estándar I. Prueba del músculo pectoral. La flecha ilustra la dirección de la fuerza del examinador contra la resistencia del paciente. El músculo deltoideo posterior trabaja como el antagonista

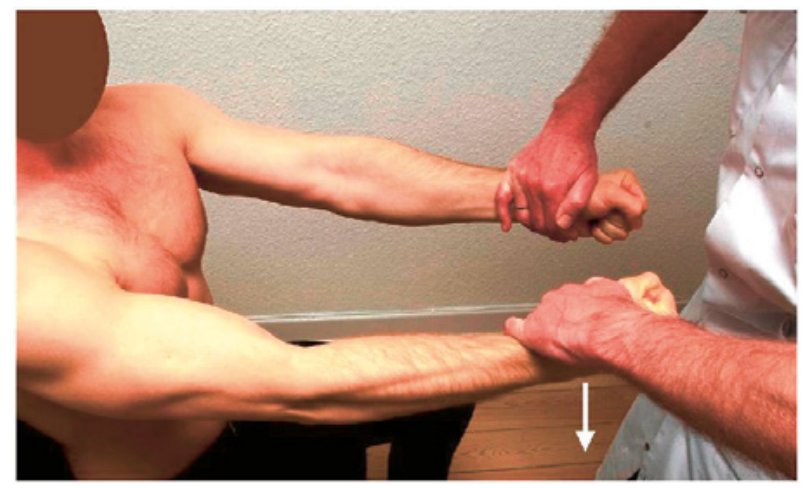

Figura 3. Postura estándar I. Prueba del músculo deltoideo posterior. La flecha ilustra la dirección de la fuerza del examinador contra la resistencia del paciente. El músculo pectoral trabaja como el antagonista

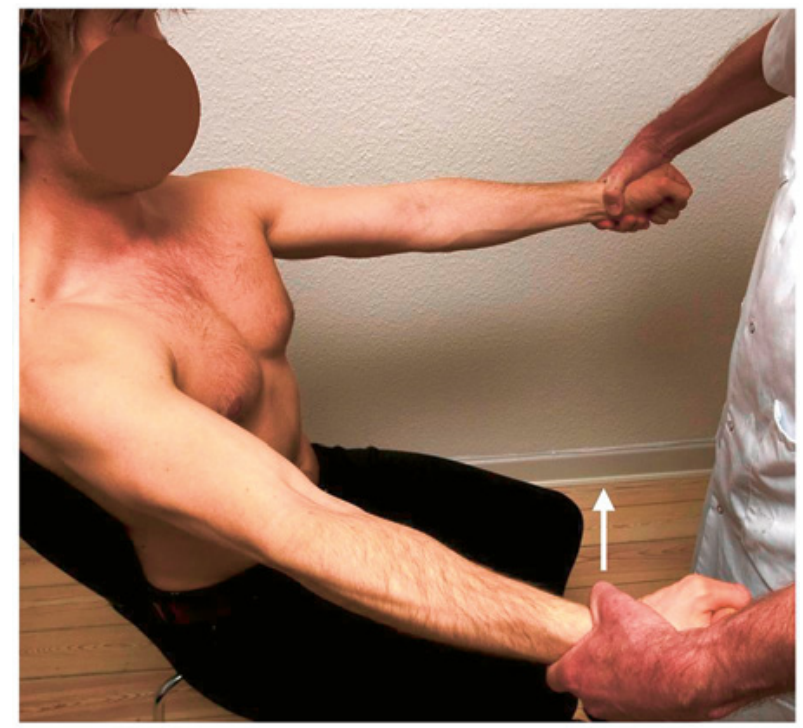


Figura 4. Postura estándar II. Prueba del músculo tríceps braquial. La flecha ilustra la dirección de la fuerza del examinador contra la resistencia del paciente. El músculo bíceps funciona como el antagonista

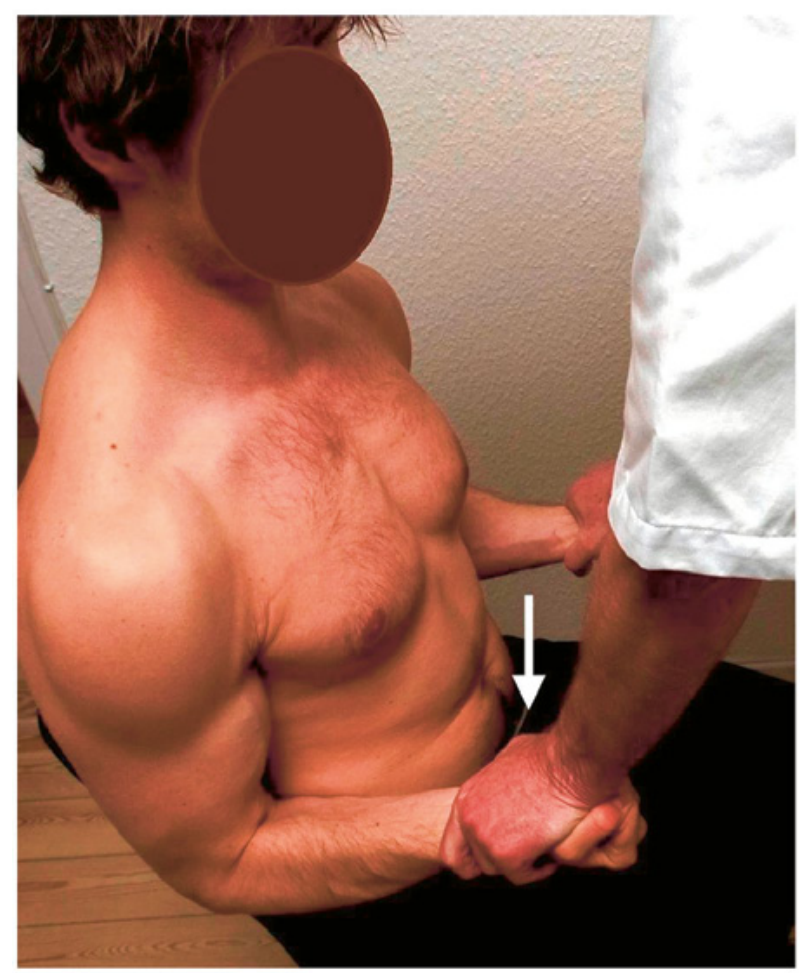

Figura 5. Postura estándar II. Prueba del músculo del bíceps braquial. La flecha ilustra la dirección de la fuerza del examinador contra la resistencia del paciente. El músculo del tríceps trabaja como el antagonista

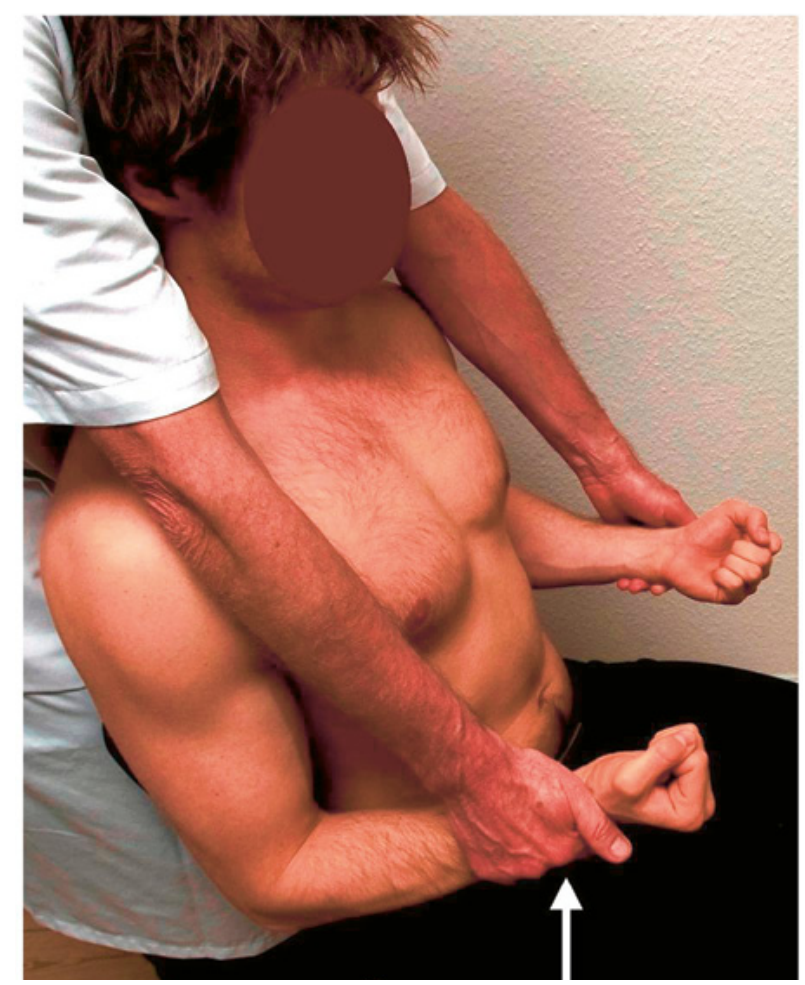


Figura 6. Posición estándar III. Prueba del músculo flexor radial del carpo. La flecha ilustra la dirección de la fuerza del examinador contra la resistencia del paciente. El extensor radial corto de la muñeca trabaja como el antagonista

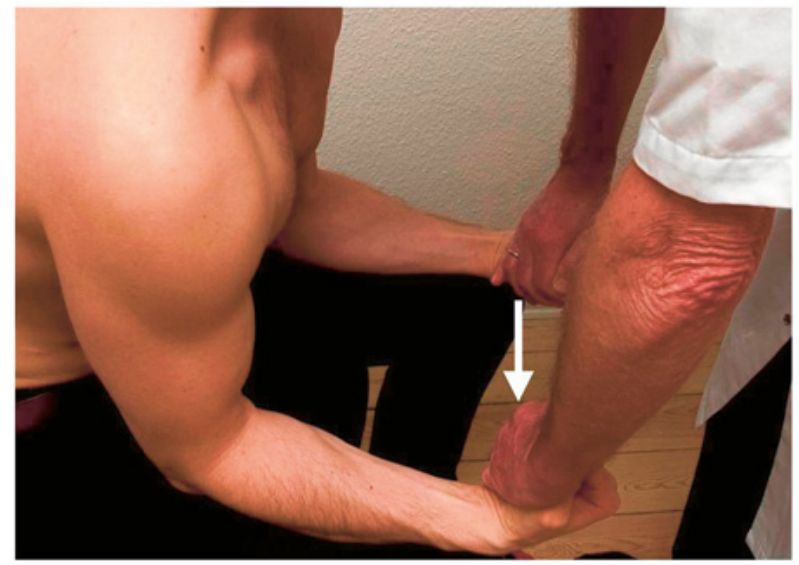

Figura 7. Postura estándar III Prueba del músculo extensor radial corto del carpo. La flecha ilustra la fuerza del examinador contra la resistencia del paciente. El músculo flexor radial del carpo trabaja como antagonista

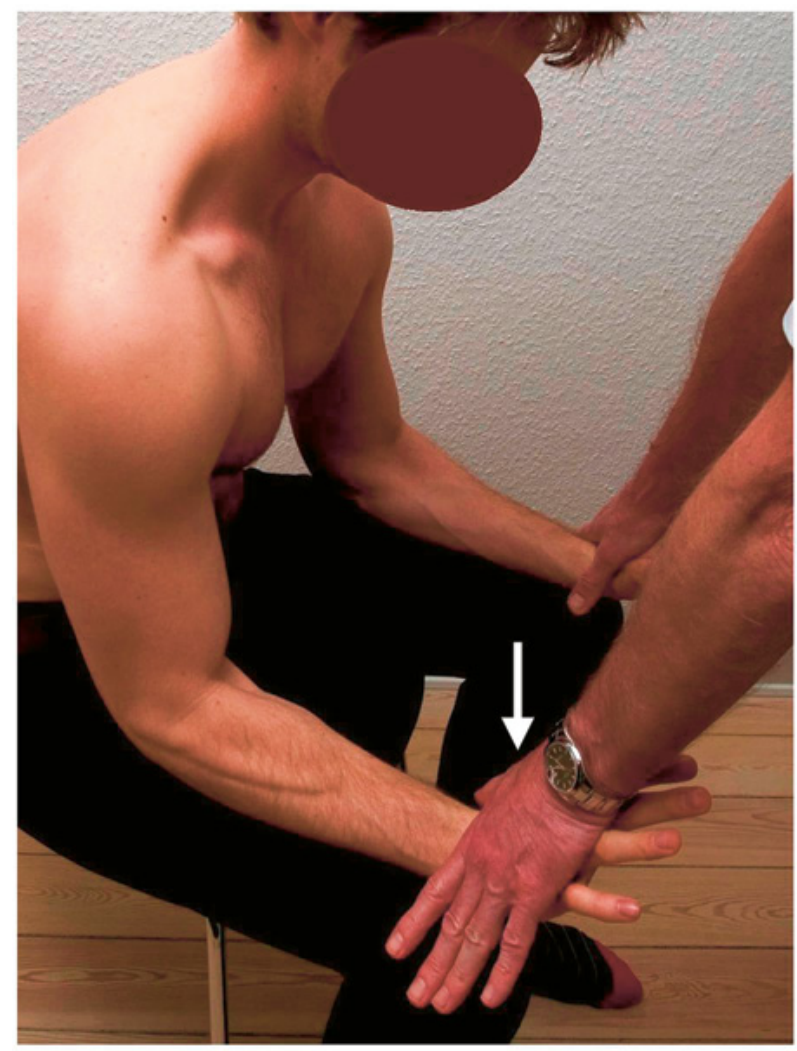

\section{Estadística}

La coincidencia entre los examinadores con relación a la identificación de la debilidad(es) muscular individual y los patrones de debilidad(es) fue evaluado por la estadística kappa de Cohen. Las mismas estadísticas se aplicaron para estimar la relación de la presencia de algún patrón o patrones con el criterio convencional (dolor, debilidad y/o entumecimiento/hormigueo). 
Tabla II. Fiabilidad entre calificadores de la identificación de debilidad en 6 músculos por parte de los examinadores

\begin{tabular}{ccccc}
\hline & \multirow{2}{*}{$\begin{array}{c}\text { Alguna } \\
\text { debilidad }\end{array}$} & \multicolumn{2}{c}{ Examinador 2 } & \multirow{2}{*}{ Total } \\
\cline { 3 - 4 } & Ausente & Presente & \\
\hline \multirow{2}{*}{ Examinador 1} & Ausente & $\mathbf{2 3}$ & 11 & 34 \\
\cline { 2 - 5 } & Presente & 5 & $\mathbf{4 3}$ & 48 \\
\hline Total & & 28 & 54 & 82 \\
\hline
\end{tabular}

La estadística Kappa de Cohen es una medida para testear si la coincidencia entre calificadores de datos categóricos exceden los niveles de posibilidad: kappa $=\left(\mathrm{p}_{\mathrm{o}}-\mathrm{p}_{\mathrm{e}}\right) /$ $\left(1-\mathrm{p}_{\mathrm{e}}\right)$ donde $\mathrm{p}_{\mathrm{o}}$ es la proporción de la coincidencia observada; $\mathrm{y}_{\mathrm{e}}$ es la proporción de la coincidencia esperada por la posibilidad. El coeficiente-kappa tiene un máximo de 1.0 $\mathrm{y}$ es interpretado como kappa: $<0.2=$ pobre, $0.21-0.40=$ discreto, $0.41-0.60=$ moderado, $0.61-0.80=$ bueno, $0.81-1.00=$ muy bueno (12). Además, se calculó la sensibilidad y especificidad del enfoque en términos de la habilidad de la debilidad identificada para predecir la presencia de síntomas

\section{RESULTADOS}

\section{Fiabilidad entre calificadores}

La prueba manual de cada uno de los seis músculos individuales seleccionados fue confiable. La coincidencia relativa de la mediana y la mediana del valor-kappa para los seis músculos individuales fueron del $82 \%$ (rango 77-87\%) y 0.58 (rango 0.46-0.72) respectivamente (Tabla I).

Los dos examinadores identificaron debilidad en uno o más músculos en 48 y 54 miembros respectivamente con una coincidencia sobre la presencia o ausencia de debilidad en 43 y 23 miembros respectivamente y no coincidieron en 16 miembros (Tabla II). La resultante de la fiabilidad entre calificadores fue moderada $(80 \%$ de coincidencia relativa, kappa $=0.59$, CI 0.44-0.77).

\section{Correlación entre la presencia de síntomas y la identificación de debilidad por parte de los examinadores}

Para uno de los examinadores, el examen de los seis músculos presentó una buena correlación entre la identificación de alguna debilidad y la presencia de síntomas (kappa $=0.61$, CI $0.45-0.78)$, mientras que el otro examinador sólo alcanzó una correlación discreta (kappa $=0.33$, CI $0.13-0.53$ ). Los dos examinadores encontraron debilidad en uno o más músculos en 35 y 32 respectivamente de los 38 miembros sintomáticos. No se encontró debilidad en 31 y 22 respectivamente de los 44 miembros asintomáticos. Por lo tanto, la sensibilidad diagnóstica de la evaluación por parte de cada examinador fue de 0.92 y 0.84 respectivamente y la especificidad 0.70 y 0.50 , respectivamente. En esta muestra, los valores predictivos positivo/negativo con respecto a los síntomas fueron de 0.73/0.91 respectivamente, para un examinador y $0.59 / 0.79$ para el otro (Tabla III).

\section{Correlación de los síntomas con coincidencia entre los examinadores}

En $79 \%$ de los miembros con una valoración unánime de presencia o ausencia de debilidad por parte de los dos examinadores, la valoración coincidió con la presencia de síntomas y el valor-kappa por lo tanto, fue calculado en 0.57 (CI 0.37 - 0.77). La sensibilidad de la conclusión unánime de la presencia de debilidad en miembros sintomáticos fue 0.84 , mientras que la especificidad de identificar ausencia de debilidad en miembros 
asintomáticos fue de sólo 0.45 . Los valores predictivos positivo y negativo fueron 0.74 y 0.87 , respectivamente (Tabla IV).

\section{DISCUSIÓN}

El examen neurológico, en particular la evaluación de la función muscular individual, se ha visto desfavorecido porque muchos asumen que es «subjetivo" y por lo tanto no confiable. A pesar de la poca evidencia para este punto de vista, muchos de los pacientes frecuentemente afectados de gravedad que se quejan del dolor en miembros superiores, entumecimiento y/o hormigueo son escépticos y tienen una tendencia a rechazar el origen somático de los síntomas ${ }^{13-16}$. Esto en particular, puede aplicarse a muchos de los pacientes que presentan quejas de miembros superiores relacionadas al trabajo que no pueden ser clasificadas de acuerdo a las prácticas actuales de examinación y criterios de diagnóstico.

Se ha demostrado que la examinación exhaustiva presentada anteriormente de los puntos neurológicos seleccionados para representar la función de los nervios de los miembros superiores ha sido precisa y capaz de predecir con exactitud la presencia de síntomas $^{6-8}$ (Tabla IV). A pesar de representar un enfoque convencional que debe incluirse en el examen físico de miembros superiores, la parte neurológica del examen rara vez es aplicada con un gran nivel de detalles y puede considerarse complicada de realizar e interpretar.

Tabla III. Síntomas en miembros superiores relacionados con la identificación de alguna debilidad en seis músculos

\begin{tabular}{lccccccc}
\hline \multirow{2}{*}{$\begin{array}{c}\text { Síntomas en } \\
\text { miembros } \\
\text { superiores }\end{array}$} & $\begin{array}{c}\text { No se } \\
\text { identificó } \\
\text { debilidad }\end{array}$ & $\begin{array}{c}\text { Alguna } \\
\text { debilidad } \\
\text { presente }\end{array}$ & Kappa & $\begin{array}{c}\text { No se } \\
\text { identificó } \\
\text { debilidad }\end{array}$ & $\begin{array}{c}\text { Alguna } \\
\text { debilidad } \\
\text { presente }\end{array}$ & Kappa & Total \\
\hline Ausente & 31 & 13 & 0.61 & 22 & 22 & 0.33 & 44 \\
\hline Presente & 3 & 35 & & 6 & 32 & & 38 \\
\hline Total & 34 & 48 & & 28 & 54 & & 82 \\
\hline
\end{tabular}

Hubo muchas razones para estudiar la viabilidad de limitar el examen neurológico de miembros superiores a la evaluación de fuerza de sólo seis músculos de los miembros superiores. Son desafiantes las dificultades obvias del diagnóstico con las que los clínicos se enfrentan cuando se encuentran con pacientes que presentan quejas relacionadas con los miembros superiores, en particular los pacientes que no pueden ser clasificados de acuerdo a los criterios convencionales de diagnóstico y también acerca del uso injustificado y generalizado de etiquetas de diagnóstico que no reflejan el tejido dañado y tampoco su localización y patología. Sin embargo, también genera preocupación abogar por un examen neurológico exhaustivo que los clínicos pueden encontrar muy difícil o que requiere de mucho tiempo.

Para afrontar este desafío, el objetivo fue presentar un examen simple de ítems neurológicos por medio del cual la identificación de signos neurológicos pudiese complementar el examen físico convencional de miembros superiores y con un alto grado de certeza contribuir al diagnóstico, explicando los síntomas que podrían deberse a una condición relacionada con los nervios.

La prueba manual de solo tres pares antagonistas de músculos que trabajan sobre el hombro, el codo y la muñeca, es rápida y fácil de recordar. La interpretación del resultado de este examen también es relativamente simple.

La debilidad muscular fue frecuente en la muestra de pacientes estudiados y pudo identificarse con una mediana de valor-kappa de 0.58 (Tabla I). Esta fiabilidad es aceptable 
y de hecho, superior a aquella de otras partes del examen neurológico del que usualmente se dependía, por ejemplo, el signo de Babinski ${ }^{17}$.

Tabla IV. Coincidencia entre la identificación de alguna debilidad por parte de los dos examinadores y la relación con el estado de los síntomas

\begin{tabular}{lcccc}
\hline Síntomas & $\begin{array}{c}\text { Coincidencia } \\
\text { en ausencia }\end{array}$ & Desacuerdo & $\begin{array}{c}\text { Coincidencia } \\
\text { en presencia }\end{array}$ & Total \\
\hline Ausente & $20(38)$ & $13(4)$ & $11(2)$ & 44 \\
\hline Presente & $3(4)$ & $3(6)$ & $32(28)$ & 38 \\
\hline Total & 23 & 16 & 43 & 82 \\
\hline
\end{tabular}

Los números entre paréntesis representan las figuras correspondientes con la aplicación de la examinación completa [8]

La consistencia entre los dos examinadores acerca de sus hallazgos se opone a su modo de examinar pacientes. Por lo tanto, la prueba de estos seis músculos cumple con los requisitos de simplicidad y fiabilidad.

Los dos examinadores encontraron debilidad en uno o más músculos en el $92 \%$ y $84 \%$ de los 38 miembros sintomáticos respectivamente, lo que significa que este examen limitado es capaz de identificar la debilidad en casi todos los miembros sintomáticos en la muestra estudiada. Una debilidad considerable puede estar presente (20-25\%) aún cuando la resistencia externa muscular no la revela y entonces se requiere cierta cantidad de fuerza reducida para la detección por medio de la prueba manual ${ }^{18}$. En este contexto, los hallazgos presentados son notables. Con un pleno acuerdo entre calificadores, las estimaciones de los dos examinadores acerca de la presencia o ausencia de debilidad reflejaron los síntomas subjetivos moderadamente bien (kappa $=0.57$ ). En los miembros con coincidencia entre la evaluación de los dos examinadores, la sensibilidad de este examen limitado fue aún mayor que aquella en el examen exhaustivo presentado anteriormente $(0.84 \text { and } 0.73 \text {, respectivamente })^{8}$.

La debilidad identificada puede deberse a condiciones neuropáticas o no neuropáticas. Mientras que este estudio no puede distinguir entre estas, sí sugiere la presencia de una implicación de los nervios en una proporción de los miembros sintomáticos con debilidad muscular mientras una implicación nerviosa es menos probable en miembros sintomáticos con fuerza intacta en los seis músculos. En esta muestra de pacientes hubo pocas condiciones sintomáticas de miembros superiores sin un componente neuropático. Esta observación difiere de la percepción general de que los trastornos en miembros superiores relacionados con el trabajo, se hallan localizados principalmente en tendones, inserciones y músculos, etc.

Los dos examinadores también identificaron debilidad en una alta proporción (30\% y 50\%, respectivamente) en los 44 miembros asintomáticos (Tabla IV). En los miembros con coincidencia entre los dos examinadores, la especificidad del examen limitado a los seis músculos fue sólo del 0.45 , mientras que el examen exhaustivo presentado anteriormente fue mucho más específico $(0.86)^{8}$. La debilidad en miembros asintomáticos no puede explicarse pero está escasamente relacionada con la presencia de una aflicción en curso de los nervios periféricos como el atrapamiento, que probablemente sea doloroso.

La baja especificidad indica que un examen limitado a la prueba manual de seis músculos claramente no es apropiado para fines diagnósticos de confirmación y que la identificación de fuerza reducida requiere de un examen más a fondo como la evaluación de alodinia de los troncos nerviosos para identificar o descartar una aflicción nerviosa.

\section{El concepto de debilidad}

La debilidad puede ser un fenómeno objetivo y/o subjetivo. Puede ser de carácter global o limitada a uno o algunos músculos, por ejemplo, músculos que comparten 
inervación. Si el paciente experimenta debilidad o no, el fenómeno objetivo de identificar fuerza reducida en uno o más músculos individuales durante el examen físico, puede ser tema de interpretación.

La debilidad puede reflejar el estado de los músculos de un sujeto sano en mala condición física, por ejemplo, a consecuencia de la inactividad o puede estar relacionada con astenia que acompaña un trastorno que puede estar limitado o no al sistema musculoesquelético en sí mismo. La debilidad muscular también puede estimularse para lograr alguna ventaja. Lo común de estas situaciones es que la debilidad rara vez ocurre en patrones con algunos músculos débiles y otros intactos. Además, la debilidad puede ser inducida por el dolor (a pesar de que esta terminología probablemente se usa más de lo que se la justifica).

La debilidad inducida por el dolor tiende a involucrar un único músculo o algunos músculos mientras que otros músculos con la misma inervación generalmente pueden testearse sin ser agravados por el dolor.

La debilidad individual o patrones de debilidad no necesariamente se deben a paresias, es decir, una aflicción del sistema nervioso periférico (o central). La debilidad global (en este caso en los seis músculos examinados) es rara pero si está presente puede representar una aflicción de todas las cuerdas del plexo braquial o puede ser el resultado de las causas que se enumeran anteriormente. La debilidad en un único músculo puede también tener otras causas diferentes a una aflicción del nervio que inerva a ese músculo. El uso doloroso de algún músculo debido a una tendinitis por ejemplo, puede evitar el esfuerzo de la fuerza plena y va a estar acompañado por dolor de la estructura. Sin embargo, las debilidades de varios músculos con un patrón en conformidad con hechos anatómicos (tales como patrones de inervación de un nervio periférico) tienen más probabilidades de representar paresias y reflejar una condición neurológica en lugar de no neurológica. Además, la identificación de alodinia mecánica a lo largo del tronco nervioso, adonde de acuerdo con el patrón de debilidad la aflicción nerviosa puede estar localizada, sugeriría que la debilidad está relacionada con una condición neuropática focal.

Un patrón de paresia muscular que ocurre secundariamente a una aflicción nerviosa periférica como el atrapamiento, probablemente esté acompañado de alodinia mecánica de los troncos nerviosos en el lugar del atrapamiento ${ }^{7}$. La ausencia de dolor en los troncos nerviosos, por otro lado, se opone a la teoría de que el atrapamiento de nervios en curso está causando la debilidad. De la misma manera, la debilidad muscular acompañada de alteraciones de la sensibilidad, probablemente represente una paresia, si el músculo débil y la sensibilidad cutánea alterada tienen la misma inervación. En consecuencia, la demostración de alodinia del tronco nervioso o disfunción sensorial con una localización apropiada además de la debilidad, va a incrementar la especificidad comparada con la del examen aislado de fuerza muscular.

En un estudio previo con la misma muestra de pacientes, hubo una destacada sensibilidad del tronco nervioso en localizaciones específicas que se relacionaron con los patrones de debilidad descubiertos ${ }^{7}$. Este hallazgo sugiere que las debilidades identificadas representan paresia. Por ejemplo, la alodinia mecánica estuvo presente en el plexo braquial en el surco deltoide-pectoral en todos los 14 miembros en los que ambos examinadores identificaron un patrón de debilidad (deltoideo posterior, bíceps, flexor radial del carpo de la muñeca) de acuerdo con una plexopatía braquial infraclavicular (síndrome del pectoral menor) ${ }^{7}$. Por lo tanto, la evaluación de la alodinia del tronco nervioso además de la prueba de fuerza muscular mejoraría la especificidad.

\section{Patrón para la comparación}

Lo más probable es que las paresias sean sintomáticas (validez convergente), mientras que los síntomas van a ser relativamente menos probables en miembros sin paresias (validez discriminante). Esta lógica se aplica a pesar de reconocer que los 
síntomas pueden tener otras causas diferentes a una aflicción o aflicciones nerviosas y que éstas pueden ser asintomáticas. Aunque se reconoce que los síntomas no son un criterio ideal para la comparación, no es posible aplicar un criterio mejor en este estudio. No existe una norma de oro $^{8}$.

Para que la electrofisiología sirva como norma de oro requeriría una evaluación global de la conducción nerviosa en muchos niveles en un gran número de nervios así como estudios electrofisiológicos de múltiples músculos. Dicha examinación exhaustiva bilateral de los nervios de miembros superiores, el plexo braquial y las raíces sería dolorosa, costosa y demandaría mucho tiempo y por estas razones, no sería viable. Más importante aún, la electrofisiología es tema de interpretación y por razones técnicas y otras, no puede servir como estimación válida para la neuropatía focal ${ }^{19}$. Por ejemplo, la compresión del nervio mediano a nivel del codo (síndrome del pronador) rara vez se detecta midiendo la velocidad de la conducción sensorial y motriz ${ }^{20,21}$.

El diagnóstico electrofisiológico del síndrome del túnel radial tampoco es confiable ${ }^{22}$. La plexopatía braquial constituye un enorme desafío con respecto al electrodiagnóstico ${ }^{23-26}$. La confianza infundada en la evaluación electrofisiológica de los atrapamientos de nervios como éstos pueden prevenir un diagnóstico correcto por no poner énfasis en los parámetros neurológicos físicos tales como los aplicados en este estudio y en anteriores ${ }^{6,7}$. Las limitaciones comparables se aplican a los estudios de imagen. Mientras los nervios y anomalías circundantes pueden visualizarse ${ }^{27}$, la precisión con respecto a la detección de, por ejemplo, atrapamiento de nervios por ultrasonido e imágenes por resonancia magnética sigue siendo desconocida. Esto se debe a que el resultado de la imagen se comparó con estándares, tales como hallazgos quirúrgicos o electrofisilogía ${ }^{28}$ que no sirven como estimaciones válidas para estas condiciones. Un intento por hacer eso puede causar además un riesgo de confundir por indicación.

\section{Interpretación}

La debilidad muscular de acuerdo con la inervación sugiere al examinador que el paciente es confiable, mientras que la presencia de debilidad con una distribución aleatoria debe ser seriamente examinada. La interpretación del resultado de la prueba muscular fue proporcionada por Hagert y Hagert ${ }^{5}$. Con una fuerza normal en los seis músculos seleccionados para este estudio, muchas localizaciones de neuropatía serían improbables, por ejemplo, el plexo braquial, el nervio axilar, el nervio radial y el nervio mediano a nivel del codo. Por otra parte, si la debilidad en cualquiera de estos músculos no puede ser explicada de manera satisfactoria desde otro razonamiento en un miembro sintomático, el clínico no debe concluir, sin cuestionar, que hay ausencia de neuropatía.

La identificación de toda debilidad muscular debe estar seguida de una examinación más completa de los nervios de los miembros superiores para brindar más evidencia en la definición y localización de algún tipo de aflicción nerviosa, por ejemplo, una prueba de músculos adicionales y una evaluación de nuevos puntos neurológicos. En particular, debe buscarse alodinia mecánica del tronco nervioso ${ }^{6-8}$. Con debilidad en alguno de los seis músculos, la presencia aislada del síndrome del túnel carpiano, neuropatía ulnar y síndrome del túnel radial es improbable. Se debe reconocer la probable presencia de doble o triple compresión ${ }^{29,30}$, que de acuerdo a nuestros estudios previos parecen ser fenómenos comunes en la muestra de pacientes estudiados con síntomas en miembros superiores ${ }^{7}$.

Este estudio presenta el resultado de la prueba de seis músculos solamente pero el total de la examinación constó de un número mayor de ítems neurológicos (fuerza en 16 músculos, sensibilidad en 7 territorios y mecanosensibilidad del tronco nervioso en 20 lugares). La examinación comenzó con la prueba manual de músculos seguida de prueba sensorial y finalmente palpación de tronco nervioso. Para evitar una evaluación tendenciosa de las pruebas musculares de otros hallazgos tales como mecanosensibilidad anómala del tronco nervioso, se suponía que los examinadores registraban todos los hallazgos independientemente uno del otro. La baja especificidad (muchos músculos 
débiles en miembros asintomáticos) se opone a dicha tendencia que, sin embargo, no puede excluirse.

\section{Limitaciones}

Este estudio se basa en el concepto de que es posible examinar músculos individuales de manera aislada sin la interferencia de otros músculos. Mientras que es viable examinar ciertos músculos de manera aislada, una correcta y completa evaluación de fuerza muscular individual no puede lograrse cuando varios músculos participan en un movimiento particular. Este estudio apunta a abordar este desafío posicionando el miembro para optimizar la función del músculo a ser evaluado y al mismo tiempo impedir la influencia de otros músculos (Tabla I).

Los seis músculos estudiados no representan la raíz Th1 ni los tres atrapamientos nerviosos comunes de miembros superiores: neuropatía ulnar a nivel del codo así como también la muñeca, síndrome del túnel carpiano y síndrome del túnel radial. La identificación de la neuropatía ulnar, síndrome del túnel carpiano y síndrome del túnel radial requiere de la examinación de la fuerza en el abductor del meñique, músculo abductor corto del pulgar y el músculo extensor cubital del carpo ulnar respectivamente ${ }^{10}$. Estas condiciones pueden ocurrir de manera aislada o acompañar una aflicción más proximal del radio o nervios medianos, el plexo braquial o las raíces. La alta sensibilidad en la muestra examinada indica la adecuación de examinar seis músculos como un enfoque diagnostico en los nervios de miembros superiores y la infrecuencia de la presencia aislada de estas tres ubicaciones comunes de atrapamiento en una muestra de pacientes derivados a un departamento de medicina laboral.

Los síntomas no son necesariamente causados por aflicciones de nervios periféricos pero pueden ser causados por trastornos en miembros superiores de carácter no neurógenos que por lo tanto también deben examinarse. Estos trastornos pueden ocurrir de manera aislada, por ejemplo, la plexopatía braquial puede complicar una tendinitis de hombro; la epicondilitis lateral o inflamación de la articulación radiohumeral puede afectar los nervios radial o interóseo posterior adyacente; el síndrome del túnel carpiano puede desarrollarse de manera secundaria al aumento de presión a causa de la inflamación de los tendones flexores en el túnel carpiano.

Los síntomas neuropáticos pueden localizarse lejos de una lesión focal, por ejemplo, el dolor en codo o muñeca puede originarse de una plexopatía braquial o de una compresión en la raíz cervical. Por lo tanto, la examinación física de miembros superiores no debe limitarse al área sintomática sino cubrir el cuello y todo el miembro.

Los clínicos tienden en principio a interpretar el dolor neuropático en miembros superiores y la disfunción como síndrome del túnel carpiano o compresión de una raíz cervical, mientras que se contempla y examina en menor grado a la implicación de la porción intermedia de la ramificación nerviosa de miembros superiores de casi un metro de largo. Teniendo en cuenta la frecuencia de la examinación de esta parte de los nervios de miembros superiores de anomalías neurológicas de acuerdo con la plexopatía braquial infraclavicular, neuropatía mediana y neuropatía interósea posterior a nivel del codo ${ }^{7}$, los autores consideran al enfoque limitado entre pacientes en medicina laboral como un gran problema.

\section{Consecuencias de los hallazgos}

La baja especificidad (0.45) de un examen limitado a seis músculos es claramente insuficiente para un diagnóstico de confirmación, mientras que la alta sensibilidad puede ser un argumento para usar el examen de estos músculos como una herramienta prediagnostica para la neuropatía de miembros superiores. Este control puede aplicarse en el ámbito clínico así como epidemiológico por ejemplo, trabajadores con riesgo de neuropatía en miembros superiores. La viabilidad del uso del examen muscular como control debe estudiarse en poblaciones expuestas con enfermedades que varían en cuanto 
a frecuencia y gravedad. La relación con el estado del síntoma del resultado de un examen cegado más exhaustivo de ítems neurológicos se ha demostrado en una muestra de operadores de computadoras «Sanos» y activos.

Este estudio no apoya la hipótesis de que la función muscular alterada no puede detectarse en pacientes con una leve compresión nerviosa ${ }^{32}$. En realidad, sugiere que el hecho de no incluir el examen de la fuerza muscular individual en la examinación física de pacientes con trastornos en miembros superiores, en particular pacientes que de otro modo serían diagnosticados no clasificables, puede tener consecuencias imprevistas. En el ámbito clínico, se puede malinterpretar a los pacientes o brindar el tratamiento incorrecto o no brindarles tratamiento. En los estudios epidemiológicos las medidas insensibles de efectos en la salud pueden causar resultados negativos erróneos y en consecuencia una prevención perdida.

\section{La neuropatía de miembros superiores como una condición relacionada al trabajo-relación con el síndrome por esfuerzo de repetición}

Este estudio no ha tenido como objetivo analizar la causalidad sino simplemente evaluar los potenciales de una simple evaluación física de la función muscular en una muestra derivada a un hospital clínica de medicina laboral.

Sin embargo, una serie de informes han tratado las aflicciones nerviosas en miembros superiores relacionadas con el trabajo ${ }^{30,32}$ incluyendo plexopatía braquial $^{23,33}$. Werner $^{34}$ y Hagert et al. ${ }^{35}$ informaron que las cargas rotacionales de antebrazo causan síndrome del túnel radial en lugar de epicondilitis y Stål et al. describieron síndrome del pronador en una elevada proporción de mujeres ordeñadoras ${ }^{36}$. Los datos epidemiológicos recientes avalan que la neuropatía de miembros superiores puede estar relacionada con el trabajo tal como se ha mostrado, por ejemplo, síndrome del túnel radial ${ }^{37}$.

\section{CONCLUSIONES}

La prueba manual de los seis músculos de miembros superiores es fácil de aprender e interpretar y rápida de realizar. Se ha demostrado que esta examinación también es confiable y que el resultado del examen refleja los síntomas.

En la muestra de pacientes estudiados derivados al departamento de medicina laboral, se ha demostrado que la prueba manual de los seis músculos de miembros superiores es un enfoque altamente sensible en la identificación de debilidad que puede estar relacionada a aflicciones nerviosas. Debido a la limitada especificidad, sin embargo, la examinación no es apropiada para un diagnóstico confirmativo. Puede servir para un enfoque de diagnóstico preliminar para neuropatía de miembros superiores en pacientes individuales así como también en poblaciones. La examinación puede resultar particularmente útil cuando el examen físico convencional de miembros superiores no puede explicar o no puede explicar completamente las quejas del paciente.

Si es positiva, esta examinación debe estar seguida de una nueva evaluación neurológica. Si es negativa, el examinador debe aún considerar la probable presencia de condiciones neuropáticas que este examen no cubre, en particular la neuropatía ulnar, el síndrome del túnel radial y el síndrome del túnel carpiano.

Los hallazgos presentados pueden sostener que, en la examinación física, los clínicos que tratan con trastornos en miembros superiores deben incluir una evaluación manual de la fuerza muscular en seis músculos de miembros superiores que representan tres pares antagonistas: pectoral mayor/deltoideo posterior, bíceps/tríceps y flexor radial de la muñeca/extensor radial corto de la muñeca. 


\section{Conflictos de interés}

Los autores declaran que no tienen conflictos de interés.

\section{Agradecimientos}

El professor C-G Hagert, Lund, ha desarrollado el enfoque para el diagnóstico físico relativo a la prueba sistemática de músculos individuales y la identificación de mialgia en probables ubicaciones de la neuropatía. Es reconocido por brindar su conocimiento y experiencia en este campo y por la enseñanza de la prueba muscular manual.

Se ha recibido apoyo financiero de Statens Sundhedsvidenskabelige Forskningsråd, Copenhagen (Grant nr. 9702593), Den Samfundsvidenskabelige Forskningsfond, Ringkøbing (Grant nr. 2-44-4-18-97), y Lida \& Oskar Nielsens Fond, Esbjerg.

\section{REFERENCIAS BIBLIOGRÁFICAS}

1. Marx RG, Bombardier C, Wright JG: What do we know about the reliability and validity of physical examination tests used to examine the upper extremity? J Hand Surg [Am] 1999; (24):185-193.

2. Katz JN, Stock SR, Evanoff BA, Rempel D, Steven MJ, Franzblau A, Gray RH: Classification criteria and severity assessment in work-associated upper extremity disorders: Methods matter. Am J Ind Med 2000; (38): $369-372$.

3. Palmer K, Cooper C: Repeated Movement and Repeated Trauma Affecting the Musculoskeletal Disorders of the Upper Limbs. In Hunter's Diseases of Occupations. Edited by Baxter P, Adams P, Aw T, Cockcroft A, Harrington J. London: Arnold; 2000:453-475.

4. Coggon D, Martyn C, Palmer KT, Evanoff B: Assessing case definitions in the absence of a diagnostic gold standard. Int J Epidemiol 2005; (34):949-952.

5. Hagert C-G, Hagert E: Manual Muscle Testing - A Clinical Examination Technique for Diagnosing Focal Neuropathies in the Upper Extremity. In Upper Extremity Nerve Repair - Tips and Techniques: A Master Skills Publication. Edited by Slutsky DJ. 6300, North River Rd. Suite 600, Rosemont, IL 60018-4256: American Society for Surgery of the Hand; 2008:451-465.

6. Jepsen J, Laursen L, Larsen A, Hagert CG: Manual strength testing in 14 upper limb muscles. A study of the inter-rater reliability. Acta Orthop Scand 2004; (75):442-448.

7. Jepsen JR, Laursen LH, Hagert C-G, Kreiner S, Larsen AI: Diagnostic accuracy of the neurological upper limb examination I. Inter-rater reproducibility of findings and patterns. BMC Neurol 2006; 6:8.

8. Jepsen JR, Laursen LH, Hagert C-G, Kreiner S, Larsen AI: Diagnostic accuracy of the neurological upper limb examination II. The relation to symptoms of patterns of findings. BMC Neurol 2006; 6:10.

9. Hagert C-G: Clinical Assessment of the Upper Limb Nerve Tree. Malmö: Scandinavian Society for Surgery of the Hand, Autumn Meeting; 1993:50.

10. Jepsen JR, Hagert C-G: Muscle testing in the diagnosis of work-related upper limb complaints. Europ Neurol J 2010; (4):73-81.

11. The Editorial Committee for the Guarantors of Brain: Aids to the Examination of the Peripheral Nervous System. London: Ballière Tindall; 1986:1-61.

12. Altman DG: Some Common Problems in Medical Research. In Practical Statistics for Medical Research. London: Chapman \& Hall; 1992:409-419.

13. Quintner JL, Elvey RL: Understanding "RSI»: A review of the role of peripheral neural pain and hyperalgesia. J Man Manipul Ther 1993; (1):99-105.

14. Quintner JL, Cohen ML, Burvill PW: Occupation neuroses and the psychogenic connotation of 'repetition strain injury': The misconstruction of neurosis. Integr Psychiatry 1994; (10):165-184.

15. Gibson SJ, LeVasseur SA, Helme RD: Cerebral event-related responses induced by CO2 laser stimulation in subjects suffering from cervico-brachial syndrome. Pain 1991; (47):173-182.

16. Helme RD, LeVasseur SA, Gibson SJ: RSI revisited: evidence for psychological and physiological differences from an age, sex and occupation matched control group. Aust New Zeal J Med 1992; (22):23-29.

17. Miller TM, Johnston SC: Should the Babinski sign be part of the routine neurologic examination? Neurology 2005; (65):1165-1168. 
18. Wadsworth CT, Krishnan R, Sear M, Harrold J, Nielsen DH: Intrarater reliability of manual muscle testing and hand-held dynametric muscle testing. Phys Ther 1987; (67):1342-1347.

19. Krarup C: Pitfalls in electrodiagnosis. J Neurol 1999; 246:1115-1126.

20. Hartz CR, Linscheid AL, Gramse RR, Daube JR: The pronator teres syndrome: Compression neuropathy of the median nerve. J Bone Joint Surg Am 1981; (63):885-890.

21. Buchthal F, Rosenfalck A, Trojaborg W: Electrophysiological findings in entrapment of the median nerve at wrist and elbow. J Neurol Neurosurg Psychiatry 1974; (37):340-360.

22. Plate AM, Green SM: Compressive radial neuropathies. Instr Course Lect 2000; (49):295-304.

23. Mackinnon SE, Novak CB: Evaluation of the patient with thoracic outlet syndrome. Semin Thor Cardiovasv Surg 1996; (8):190-200.

24. Rousseff R, Tzvetanov P, Valkov I: Utility (or futility?) of electrodiagnosis in thoracic outlet syndrome. Electromyogr Clin Neurophysiol 2005; (45):131-133.

25. Parziale JR, Akelman E, Weiss AP, Green A: Thoracic outlet syndrome. Am J Orthop 2000; (29):353-360.

26. Komanetsky RM, Novak CB, Mackinnon SE, Russo MH, Padberg AM, Louis S: Somatosensory evoked potentials fail to diagnose thoracic outlet syndrome. J Hand Surg [Am] 1996; (21):662-666.

27. Kim S, Choi JY, Huh YM, Song HT, Lee SA, Kim SM, Suh JS: Role of magnetic resonance imaging in entrapment and compressive neuropathy-what, where, and how to see the peripheral nerves on the musculoskeletal magnetic resonance image: part 2. Upper extremity. Eur Radiol 2007; (17):509-22.

28. Zaidman CM, Seelig MJ, Baker JC, Mackinnon SE, Pestronk A: Detection of peripheral nerve pathology: comparison of ultrasound and MRI. Neurology 2013; (80):1634-1640.

29. Mackinnon SE: Double and multiple "crush" syndromes. Double and multiple entrapment neuropathies. Hand Clin 1992; (8):369-390.

30. Novak CB, Mackinnon SE: Multilevel nerve compression and muscle imbalance in work-related neuromuscular disorders. Am J Ind Med 2002; (41):343-352.

31. Jepsen JR, Thomsen G: A cross-sectional study of the relation between symptoms and physical findings in computer operators. BMC Neurol 2006; 6:40.

32. Novak CB, Mackinnon SE: Evaluation of nerve injury and nerve compression in the upper quadrant. $J$ Hand Ther 2005; (18):230-240.

33. Pascarelli EF, Hsu YP: Understanding work-related upper extremity disorders: clinical findings in 485 computer users, musicians, and others. J Occup Rehabil 2001; (11):1-21.

34. Werner CO: Lateral elbow pain and posterior interosseus nerve entrapment. Acta Orthop Scand 1979; (Suppl.174):1-62.

35. Hagert C-G, Lundborg G, Hansen T: Entrapment of the posterior interosseous nerve. Scand J Plast Reconstr Hand Surg 1977; (11):205-212.

36. Stål M, Hagert C-G, Moritz U: Upper extremity nerve involvement in Swedish female machine milkers. Am J Ind Med 1998; (33):551-559.

37. Roquelaure Y, Raimbeau G, Dano C, Martin Y-H, Pelier-Cady C, Mechali S, Benetti F, Mariel J, Fanello S, Penneau-Fontbonne D: Occupational risk factors for radial tunnel syndrome in industrial workers. Scand $J$ Work Environ Health 2000; (26):507-513. 